\title{
The Simulation of Switched Reluctance Motor with the Rapid Nonlinear Method
}

\author{
Zhen Guo, Min Zhang \& Xinping Ding \\ School of Automation Engineering, QingDdao Technological University \\ Tel: 86-532-8878-2001Ｅ-mail: qs2004b@163.com
}

\begin{abstract}
Considering the significant magnetic saturation and the double salient poles of Switched Reluctance Motor (SRM), this paper presents a new method combining the Rapid Nonlinear Simulation Method with the Equivalent Magnet Circuit Analysis Model, which can make the accuracy and rapidity of the calculation of the performance of SRM achieved. This paper also presents a model of SRM, implements the rapid nonlinear simulation method to the model and lay the necessary foundation for SRM optimization.
\end{abstract}

Keywords: SRM, Equivalent Magnet Circuit Analysis, Rapid Nonlinear Simulation

\section{Instruction}

The switched reluctance motor (SRM) is simple, low cost, fault tolerant, and has simple converter and control requirements. The motor is suitable for many variable-speed and servo-type applications and is gaining increasing attention in the motor drive industry. However, the nonlinear magnetization characteristics of the SRM is one of the main disadvantages, which produced by piecewise first- or second-order function of flux linkage against rotor position. There are many ways to analyze the nonlinear magnetization characteristics, such as nonlinear magnet parameter analysis, rapid nonlinear theory analysis, equivalent magnet circuit analysis model, inductance parameter analysis and so on. The method of Nonlinear magnet parameter analysis is very accurate, but with great computation load and it only can be used to analyze the situation when one phase is in work; the algorithms of the rapid nonlinear theory analysis are extremely fast, but not enough accurate; performance characteristic can be analyzed by the inductance parameter method, however, increment inductance or average inductance is the function of winding current and rotor position, it is very difficult to calculate the inductance accurately; the precision of the equivalent magnet circuit analysis model is high and it can be applied to analyzed the situation when many phases are performed, but the disadvantage of this way is that it inquired greater computation. If more accurate mathematical model is established, it will result in greater computation and will slow the calculation speed due to significant nonlinear magnetization characteristics of SRM. So a better method should be found to establish mathematical model which not only own more precision character but also greater calculation speed. This paper presents a new method combining the rapid nonlinear simulation method with the equivalent magnet circuit analysis model. It can make the accuracy and the rapidity of the calculation of the performance of switched reluctance motor achieved and laid the necessary foundation for SRM optimization.

\section{The Foundation of Equivalent Magnet Circuit Analysis Model of SRM}

Phase linkage is concerned when establishing the equivalent magnet circuit analysis model and variable structure model and it is proposed in article (Miller T J E, McGilp M. 1990). With the introduction of "overlapping unit and equivalent magnet circuit"(F.Filicori et al. 1993), equivalent magnet circuit analysis model four-phase motor is found. The parameters of the model are defined in Figure 1.

$G_{1} \sim G_{4}$ are the equivalent permeances of the overlapping unit, we can obtain them as follows:

$G_{i}=\Phi(F, \theta) / F \quad(i=1,2,3,4)$

$\Phi(F, \theta)$ is equivalent magnetic flux of the overlapping unit and $F$ is the magnetic potential drop between different equivalent surface of the overlap unit. $G_{5} \sim G_{8}$ are the equivalent permeances of the stator-pole root segment,

$G_{i}=\mu_{F e} B_{P} L_{S} /\left(0.5 H_{p}\right) \quad(i=5,6,7,8)$

$\mu_{F e}$ is the magnetic permeability of core, $B_{p}, H_{p}$ are stator-pole width and stator-pole height respectively.

$L_{S}$ is the length of the core. $G_{s l}$ is the equivalent linkage permeance of the adjacent poles,

$G_{s l}=0.5 \mu_{0} H_{p} L_{s} / B_{0}$ 
$\mu_{0}$ is the magnetic permeability of air, $B_{0}$ is average width of stator slot. $\quad G_{9} \sim G_{12}$ are the equivalent permeances of the stator yoke end respectively,

$$
G_{i}=\mu_{F e} A_{c} / L_{c} \quad(i=9,10,11,12)
$$

$A_{c}$ is equivalent sectional area of stator yoke end, $L_{c}$ is the length of stator yoke end. $F_{1} \sim F_{4}$ are magnetic potentials of stator concentrated winding,

$$
F_{k}=N I_{k} \quad(k=1,2,3,4)
$$

$N$ is the number of turns of concentrated winding, $I_{k}$ is phase current of the number $k$.

We can get the values of phase currents, torque and power through calculation after obtaining the equivalent permeance.

\section{The Application of the Rapid Nonlinear Simulation Method to Equivalent Magnet Circuit Analysis Model of SRM}

The structure of the model is shown in Fig2 for a single curve corresponding to a fixed value of current $i$. The key points along this curve are denoted by $\xi$, with appropriate subscripts, and the corresponding rotor positions are represented by the small diagrams below (Figure 2).

Region I extends from the unaligned position $\xi_{u}\left(=\pi / N_{r}\right)$ to the 'corner point' $\xi_{1}$, where the approaching corners of stator and rotor poles are aligned. Mechanical overlap begins at $\xi_{1}$ and continues until $\theta=\xi_{a}$ at the aligned position $\left(2 \pi / N_{r}\right)$, where the curve begins its descent along a mirror image of the curve between $\xi_{u}$ and $\xi_{a}$. The overlap region is divided into regions $\mathrm{II}$ and II, with the dividing point $\xi_{1}$ and $\xi_{2}$.

The modeling in the three regions will now be described, staring with region $I$, which is simplest. When describing the region II, $\xi_{1}$ and $\xi_{h r}$ will be introduced from reference(B.K.Bose, T.J.E. Miller, 1986).

Region II:

In region $\mathrm{I}$, the straight line relationship between $\psi$ and $\theta$ is

$\psi(\theta)=\psi_{1}+k_{a}\left(\theta-\xi_{1}\right)$

and the value of $k_{a}$ can be obtained as follows,

$$
k_{a}=\frac{\psi_{h r}-\psi_{1}}{\xi_{h r}-\xi_{1}}
$$

Region I :

In order to guarantee the continuity of the curve and make it more precise, the curve is modeled by a modificatory Frohlich-like relationship:

$\psi(\theta)=\psi_{1}-\frac{A\left(\xi_{1}-\theta\right)}{B+\left(\xi_{1}-\theta\right)^{C}}$

Due to $\psi\left(\xi_{u}\right)=\psi_{u}, \psi^{\prime}\left(\xi_{u}\right)=0, \psi^{\prime}\left(\xi_{1}\right)=k_{a}$, so the answer is

$$
\begin{aligned}
& C=\frac{k_{a} \xi_{1 u}}{k_{a} \xi_{1 u}-\psi_{1 u}} \\
& B=-(1-C) \xi_{1 u}{ }^{C} \\
& A=k_{a} B
\end{aligned}
$$

Region III :

The $\psi / \theta$ curve is modeled by a second Frohlich-like equation: 
$\psi(\theta)=\psi_{h r}+\frac{A^{\prime}\left(\theta-\xi_{h r}\right)}{B^{\prime}+\left(\theta-\xi_{h r}\right)^{C^{\prime}}}$

Due to $\psi\left(\xi_{a}\right)=\psi_{a}, \psi^{\prime}\left(\xi_{a}\right)=0, \psi^{\prime}\left(\xi_{h r}\right)=k_{a}$, then the answer is

$$
\begin{aligned}
& C^{\prime}=\frac{k_{a} \xi_{a h r}}{k_{a} \xi_{a h r}-\psi_{a h r}} \\
& B^{\prime}=-\left(1-C^{\prime}\right) \xi_{a h r} C^{\prime} \\
& A^{\prime}=k_{a} B^{\prime}
\end{aligned}
$$

We can calculate the magnetization curve using the equations mentioned above after modeling different regions of the magnetization curve. The rapid nonlinear simulation method is more rapid in algorithm comparing with the finite-element techniques. We can obtain the accurate result as same as that by using the 3D finite-element techniques as long as four significant rotor positions are input correctly. Then the calculation of the equivalent magnet circuit of the overlapping unit and characteristic analysis will be accomplished.

\section{The nonlinear simulation result of the system and its analysis}

We have done the steady-state operation performance simulation using the method of combination between the rapid nonlinear simulation and the equivalent magnet circuit analysis. Chopped Current Control and Angular Position Control are two control methods of SRM. When using the Chopped Current Control, winding current is set by drive circuit, so the simulation procedure provided by this paper is applied to analyze the steady-state operation performance and we have done the calculation of the phase current, torque, power and so on. Different parameters are show as following: number of rotor poles is 6 , number of stator poles is 8 , stator outer diameter is $175 \mathrm{~mm}$, stator inner diameter is $98 \mathrm{~mm}$, air gap is $0.3 \mathrm{~mm}$, stator core length is $100 \mathrm{~mm}$, firing-angle is $-10^{\circ}$, angle of flow is $18^{\circ}$, winding resistance is $0.08 \Omega$, number of turns per phase is 90 , based on the parameters mentioned above, we get the simulation Fig 3 as follows and Fig 4 are experimental results.

From the Fig 3 and Fig 4, we know that simulation datum are close to experimental datum and calculation speed is more rapid than finite element method and accuracy is perfect, so we can say the method of combination between the rapid nonlinear simulation and the equivalent magnet circuit analysis is effective.

\section{Conclusion}

Nonlinear simulation method of steady-state operation performance is an important way to study the characters and the control methods of SRM. How to accomplish the nonlinear simulation calculation of a new project rapidly is significant to the design and optimization of SRM. This paper presents a new method that is the combining the rapid nonlinear simulation method with the equivalent magnet circuit analysis model which can make the accuracy and rapidity of the calculation of the performance of motor achieve and lay the necessary foundation for SRM optimization.

\section{References}

B.K.Bose, T.J.E. Miller. (1986). "Microcontroller control of switched reluctance motor", IEEE Transanctions on Industry Applications, Vol. IA-22, July/August 1986, pp.708-715.

F.Filicori et al. (1993). Modeling and Control Strategies for a Variable Reluctance Direct-Drive Motor. IEEE Tran.on IE-40 No.1, 1993.2

J. Ish-Shalom and D.G. Manzer. (1985). "Commutation and control of step motors," in Proc.14th symp. Incremental Motion, Contr. Syst.and Devices, June 1985,pp:283-292.

J.V.Byrne and F.Devitt. (1985). "Design and performance of a saturable variable reluctance servo motor," in Proc. Motorcon conf., Chicago, il,Oct.1985,pp.139-146.

Lawrenson P J. (1983). Switched Reluctance Motor Drives. Electronic and Power, Fed, 1983

Miller T J E, McGilp M. (1990). Nonlinear theory of the switched reluctance motor for rapid computer aided design, IEEE Proc., 1990, 137 (6, Pt. B): 337-347

R.M.Davis, W.F.Ray, and R.J.Blake. (1981). "Inverter drive for switched reluctance motor: Circuits and component ratings," IEE Proc.,vol.128, pt. B, no.2, pp. 126-136, Mar.1981. 
Tong Huai, Fu Guangiie, Huang Shenghua. Steady state simulation of a four phase hybrid step motor using the magnetic equivalent circuit model, ICEM'96, Vigo, Spain.

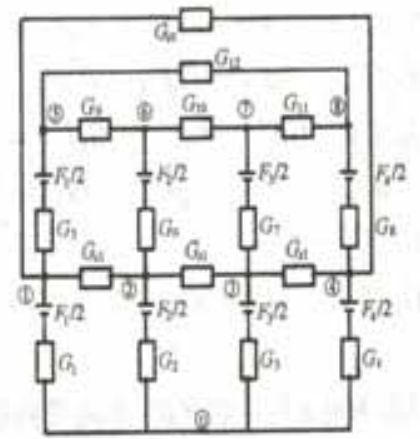

Figure 1. 8/6 SRM equivalent magnet circuit

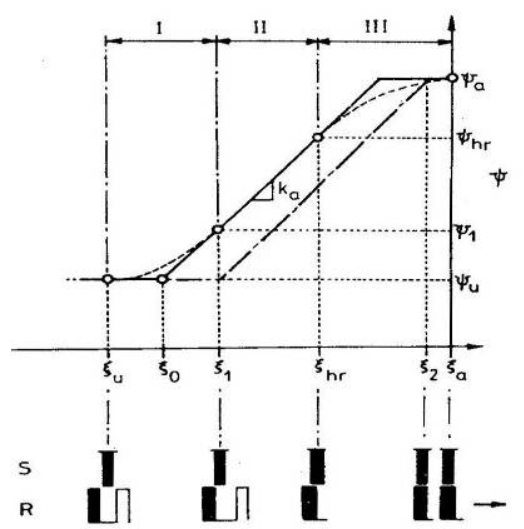

Figure 2. Modeling of a flux-linkage curve defining the domains of the three different approximation algorithms

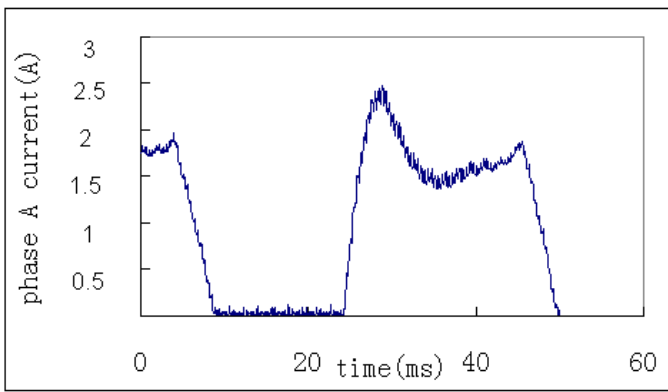

(a) Phase A current wave

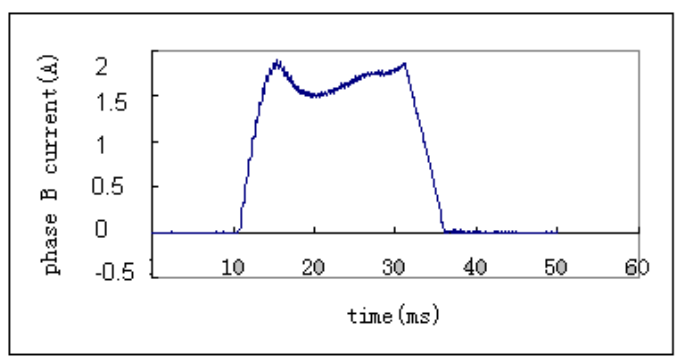

(b) Phase B current wave

Figure 3. Simulation figures of SRM 


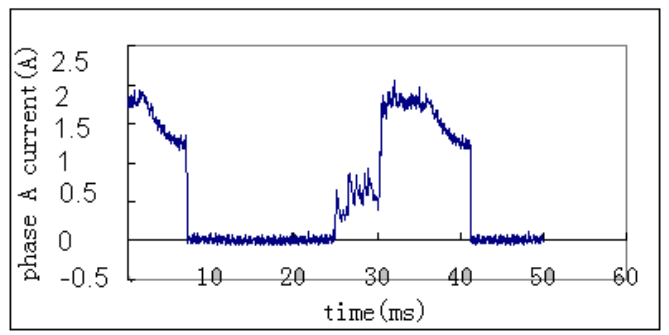

(a) Phase A current wave

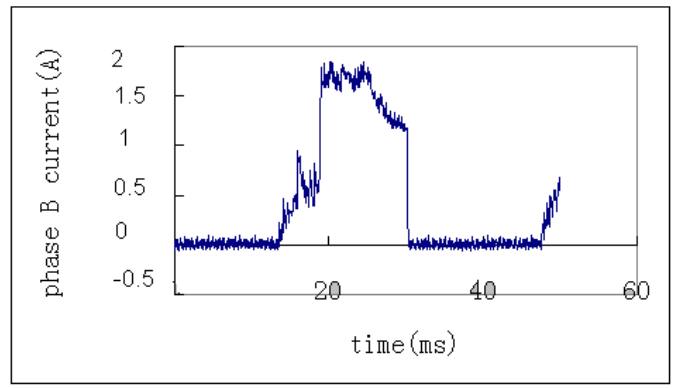

(b) Phase B current wave

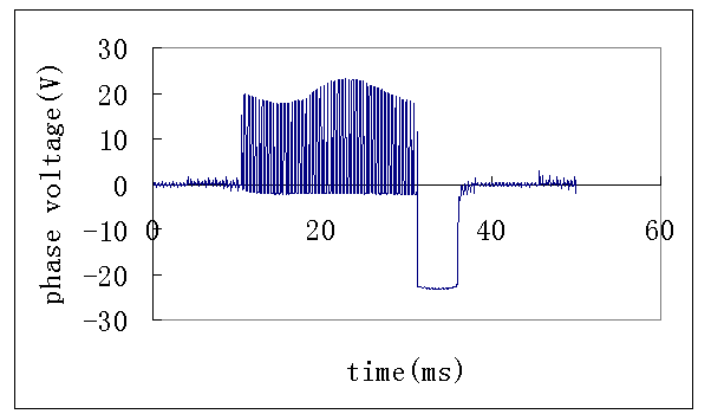

(c) Phase voltage

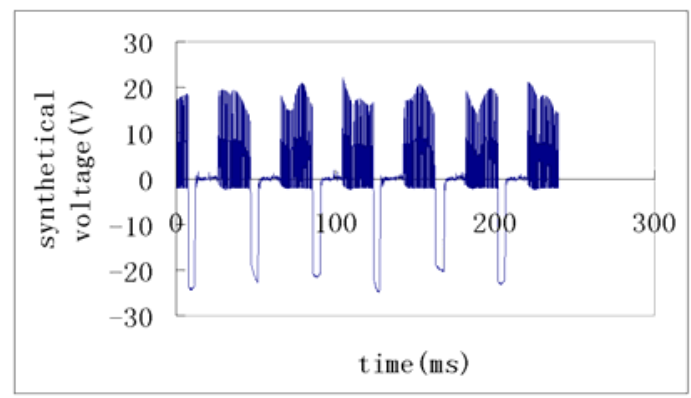

(d) Synthetic voltage

Figure 4. Experimental figures of SRM 